\title{
A unified framework for photophysical rate calculations in TADF molecules
}

\author{
Leonardo Evaristo de Sousa ${ }^{1}$ and Piotr de Silva ${ }^{1,}$ a) \\ Department of Energy Conversion and Storage, Technical University of Denmark, Anker Engelunds Vej 301, 2800 Kongens Lyngby, \\ Denmark
}

One of the challenges in organic light emitting diodes research is finding ways to increase device efficiency by making use of the triplet excitons that are inevitably generated in the process of electroluminescence. One way to do so is by thermally activated delayed fluorescence, a process in which singlet excitons undergo up-conversion to singlet states, allowing them to relax radiatively. The discovery of this phenomenon has ensued a quest for new materials that are able to effectively take advantage of this mechanism. From a theoretical standpoint, this requires the capacity to estimate the rates of the various processes involved in the photophysics of candidate molecules, such as intersystem crossing, reverse intersystem crossing, fluorescence and phosphorescence. Here we present a method that is able to, within a single framework, compute all these rates and predict the photophysics of new molecules. We apply the method to two TADF molecules and show that results compare favorably with other theoretical approaches and experimental results. Finally, we use a kinetic model to show how the calculated rates act in concert to produce different photophysical behavior.

\section{INTRODUCTION}

Organic light emitting diodes (OLEDs) have found commercial use in the last decade thanks to features such as mechanical flexibility, low production costs and tunability of its luminescence properties ${ }^{1-4}$. However, due to spin-statistics, charges injected into the active layer of an OLED tend to generate triplet and singlet excitons in a 3:1 ratio $^{5}$. Given that the efficiency of emission from triplet states is usually low, as it requires high spin-orbit couplings between triplet and ground states to occur, a cap is put on the internal efficiency of fluorescent OLED devices at $25 \%$.

In this sense, strategies to circumvent this deficiency have been developed. One approach, for instance, aimed at increasing phosphorescence efficiency by employing metal-organic complexes and taking advantage of the heavy atom effect on the spin-orbit couplings ${ }^{6,7}$. This procedure has been able to raise internal quantum efficiencies, however applications in the blue region of the spectrum become problematic due to degradation issues ${ }^{8}$ and the use of expensive materials increases the cost of device production ${ }^{9}$. Alternatively, the phenomenon of thermally activated delayed fluorescence (TADF) has been identified in the last years as a powerful means to increase the efficiency of OLED devices ${ }^{10-13}$. At the heart of this phenomenon is a monomolecular up-conversion mechanism called reverse intersystem crossing (rISC) in which triplets convert into singlet states with the aid of the available thermal energy. Evidently, the opposite intersystem crossing (ISC) - from singlet to triplet state - also takes place and more efficiently so, since it is energetically more favorable. However, if phosphorescence and non-radiative decay channels are much less efficient in comparison to rISC, the $T_{1}$ states keep converting back into $S_{1}$ until they eventually recombine either radiatively or non-radiatively. As such, a continuous recycling of triplet excitons is possible, increasing the device's quantum efficiency significantly. More recently, the combination

\footnotetext{
a)Electronic mail: pdes@dtu.dk
}

of TADF molecules with efficient organic emitters has also been proposed, giving rise to the so-called hyperfluorescencebased devices ${ }^{14,15}$.

To achieve efficient rISC, it is necessary that the energy gap between singlet and triplet states be small and that the corresponding spin-orbit coupling be non-zero. Such combination was obtained successfully in an OLED device using pure organic molecules in $2012^{16}$ by employing a molecular design strategy consisting on spatially separated donoracceptor units, which result in excited states with charge transfer character and reduced exchange interaction. However, such charge transfer excited states usually present low oscillator strengths ${ }^{17}$, which hinders the molecule's fluorescence rate. This trade-off demonstrates that new design strategies are required.

Following these results, the search for new materials that may take advantage of the TADF mechanism has become an important field of research. In this context, computational approaches play an important role, both serving as means to aid the interpretation of experimental results, but also as a tool in the development of new molecules by evaluating a compound's potential prior to synthesis ${ }^{18-20}$. To fulfill these roles, the capacity of estimating the rates of the relevant processes is essential from a theoretical point of view.

One particular important factor to consider when estimating rates for (r)ISC is the role of molecular vibrations. For instance, it has been experimentally demonstrated that intersystem crossing processes can be greatly affected by suppressing vibrational modes ${ }^{21}$, suggesting that vibronic effects must be taken into account in the description of the phenomenon. These observations have also been corroborated by different theoretical works ${ }^{22-25}$, which have suggested that vibronic couplings between different singlet and triplet states may be responsible for rISC rates that are greater than those predicted by standard first-order perturbation theory. For this reason, different methods have been used to estimate (r)ISC rates that include vibrational effects (see, for instance, Refs. 26,27 for comprehensive reviews). Among the most popular is the Marcus-Levitch-Jortner (MLJ) formalism, that has been 
widely employed for such calculations ${ }^{15,28,29}$ with mixed results. More computationally demanding approaches that involve higher order Taylor expansions of the spin-orbit coupling have also been developed ${ }^{30}$ as well as time dependent ${ }^{31}$ and quantum dynamics methods ${ }^{23}$. In spite of these various efforts, methods that are able to combine accurate results with reasonable computational cost and ease of applicability to different compounds are still wanted for.

In this work we present a temperature dependent Monte Carlo approach to rate calculations that goes beyond the Condon approximation and takes into account effects stemming from molecular vibrations. This method is based on a combination of electronic structure calculations and sampling of hundreds of molecular geometries, which helps us circumvent the calculation of vibrational overlap integrals. A similar approach has been successfully used in the past in the context of simulating absorption and emission spectra ${ }^{32,33}$ and for the estimation of singlet exciton transfer rates and diffusion lengths ${ }^{34}$. This provides us with a unified theoretical framework that allows us to calculate at once both ISC and rISC rates, as well as fluorescence and phosphorescence rates consistently. As such, the method gives us the capacity to predict the photophysics of a system and the corresponding lifetimes and relative contributions of each process to the overall timedependent decay curves.

To demonstrate the method, we apply it to two TADF molecules for which experimental rates are available: a donor-acceptor complex composed of a phenothiazine donor and a dibenzothiophene-S,S-dioxide acceptor (PTZ-DBTO2, shown in Figure 1-a) and 1,2,3,5-tetrakis(carbazol-9-yl)-4,6dicyanobenzene (4CzIPN, shown in Figure 1-b). We compare our results with other theoretical calculations, demonstrating, in particular, how the MLJ formalism fails to treat these cases. We also show that overall the different rate estimates produced with the method developed here are in agreement with experimental results. Importantly, we apply the calculated rates to a kinetic model which is able to predict the experimentally observed photophysical behavior of these molecules, highlighting the usefulness of a unified approach to rate calculations in the context of TADF molecules.

\section{METHODS}

\section{A. Rate calculations}

Here we outline the method employed for the rate calculations. A full derivation can be found in the Supporting Information (SI) file.

In general, the rate of transfer between electronic states $i$ and $f$ as a result of a perturbation term (V) applied to the Hamiltonian can be obtained by means of Fermi's Golden Rule

$$
k_{i \rightarrow f}(E)=\frac{2 \pi}{\hbar} \sum_{v, \mu} P_{i v}(T)\left|\left\langle\Psi_{i v}|V| \Psi_{f \mu}\right\rangle\right|^{2} \delta\left(E+E_{i v}-E_{f \mu}\right),
$$

\section{a}<smiles>O=S1(=O)c2ccccc2-c2cc(N3c4ccccc4Sc4ccccc43)ccc21</smiles>

b

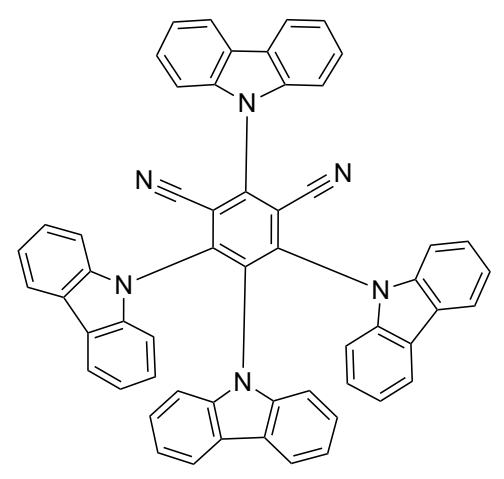

FIG. 1. Molecules analyzed in this work. a) PTZ-DBTO2 and b) 4CzIPN.

In this expression, $i(f)$ and $v(\mu)$ index electronic and vibrational levels of the initial (final) states, respectively. In addition, $P_{i v}(T)$ is the Boltzmann weighed probability of state $(i, v)$ being populated. The energy $E$ dependence of this expression refers, for instance, to photon or phonon energies that are required to ensure energy conservation in the transition process.

By recasting the above expression in the time domain and employing a set of approximations, we are able to write the rate expression as an expectation value, which can be evaluated by means of a Monte Carlo procedure as follows ${ }^{32,35}$

$$
k_{i \rightarrow f}(E)=\frac{2 \pi}{\hbar} \frac{1}{M} \sum_{k=1}^{M} V^{2}\left(\vec{R}_{k}\right) N\left(\Delta E_{f i}\left(\vec{R}_{k}\right)-E, \sigma\right) .
$$

In this expression, $M$ molecular geometries with nuclear normal coordinates $\vec{R}_{k}$ are sampled from a distribution. From these geometries, values of $V\left(\vec{R}_{k}\right)$ and $\Delta E_{f i}\left(\vec{R}_{k}\right)$ are obtained and used to calculate each argument of the summation above, in which $N\left(\Delta E_{f i}\left(\vec{R}_{k}\right)-E, \sigma\right)$ refers to a normalized gaussian function with mean $\Delta E_{f i}\left(\vec{R}_{k}\right)-E$ and standard deviation $\sigma$. The distribution used to sample molecular geometries is that for a set of harmonic oscillators at temperature $T^{36}$ : 


$$
\begin{aligned}
\rho(\vec{R}, T)=\prod_{i=1}^{3 N-6}\left(\frac{\mu_{i} \omega_{i}}{2 \pi \hbar \sinh \left(\hbar \omega_{i} / k_{b} T\right)}\right) \\
\quad \times \exp \left(-\frac{\mu_{i} \omega_{i}}{\hbar} R_{i}^{2} \tanh \left(\frac{\hbar \omega_{i}}{2 k_{b} T}\right)\right) .
\end{aligned}
$$

In this expression, $\mu_{i}$ and $\omega_{i}$ correspond to the reduced mass and angular frequency of the $i$ th normal mode, $k_{b}$ is the Boltzmann constant and $R_{i}$ are the normal coordinates. These normal modes must be obtained from frequency calculations on the initial state, that is, from $S_{1}$ optimized geometries in the case of ISC and fluorescence rate calculations and from $T_{1}$ optimized geometries in the case of rISC and phosphorescence rate estimations.

To obtain the differential emission rate for fluorescence or phosphorescence, we substitute the perturbation $V$ in Equation 2 with $^{37,38}$

$$
V_{e m i}=\frac{n^{2} E^{3} M_{i f}^{2}}{3 \pi \hbar^{3} c^{3} \varepsilon_{0}}
$$

in which $n$ is the refractive index of the medium, $\varepsilon_{0}$ is the vacuum permittivity, $c$ is the speed of light and $M_{f i}$ is the transition dipole moment between states $i$ and $f$. Importantly, as shown in the SI, computation of the transition dipole moments for phosphorescence requires the consideration of mixing between singlet and triplet states coupled by the spin-orbit perturbation.

Equation 2 provides us the fluorescence and phosphorescence spectra. The total emission $\left(k_{e m i}\right)$ rate for each phenomenon is obtained by integrating the corresponding spectrum

$$
k_{\text {emi }}=\frac{1}{\hbar} \int_{0}^{\infty} I_{\text {emi }}(E) d E
$$

In the cases of ISC and rISC, the perturbation $V$ is given by the spin-orbit coupling element $\left\langle S_{1}\left|H_{S O}\right| T_{1}>\right.$. Equation 2 provides then an expression for the (r)ISC rate as a function energy $E$. Unlike emission processes, this energy does not correspond to photon energies, but rather to phonons that can be either absorbed or emitted by the molecule. To account for this, we consider the system to be immersed in a harmonic bath that may either provide or receive the excess energy necessary for a (r)ISC process. As such, the actual rate can be obtained by means of the following convolution ${ }^{39,40}$

$$
k_{(r) I S C}=\int I_{S}(E) k_{i \rightarrow f}(\Delta E-E) d E,
$$

in which $I_{S}(E)$ is the line shape function for the low frequency solvent modes. Under the classical approximation, this term is a gaussian ${ }^{41}$

$$
I_{S}(E)=\sqrt{\frac{\pi}{\lambda k T}} \exp \left(-\frac{(\lambda+E)^{2}}{4 \lambda k T}\right)
$$

where $\lambda$ is the reorganization energy.

Performing the integration in Equation 6 finally yields

$$
\begin{aligned}
k_{(r) I S C}(E)=\frac{2 \pi}{\hbar} \frac{1}{M} & \sum_{k=1}^{M} H_{S O}\left(\left\{\vec{R}_{k}\right\}\right)^{2} \\
& \times N\left(\lambda+\Delta E_{f i}\left(\vec{R}_{k}\right), \sqrt{2 \lambda k T+\sigma^{2}}\right),
\end{aligned}
$$

\section{B. Computational Details}

Geometry optimizations and normal mode analyses were performed for both molecules in the $S_{1}$ and $T_{1}$ states. A total of 500 geometries were sampled from Equation 3 for each state. A $\sigma=k T$ factor was used for all rates and temperature was set to $300 \mathrm{~K}$. Excited state calculations were then run on all sampled geometries, from which excitation energies, transition dipole moments and spin orbit couplings were obtained. A total of 10 singlet and 10 triplet states were taken into account. The Tamm-Dancoff approximation ${ }^{45}$ (TDA) was employed throughout, as it has been demonstrated to both improve the accuracy of the excitation energies of triplet states ${ }^{46}$ and to be able to produce reliable emission spectra ${ }^{47}$. Furthermore, all calculations made use of the polarizable continuum model ${ }^{48}$ (PCM) with toluene as the solvent and were performed with the M062X functional ${ }^{49}$ along with the 6$31 \mathrm{G}(\mathrm{d}, \mathrm{p})$ basis set. The Gaussian $16^{50}$ program suite was employed for geometry optimizations and normal mode analyses whereas the QChem $5.0^{51}$ software was used for all remaining calculations.

\section{RESULTS}

The calculated values of ISC and rISC rates for PTZDBTP2 and 4CzIPN are shown in Table I. These are compared with experimental values as well as with results obtained from using two other theoretical approaches. The first one is the aforementioned Marcus-Levitch-Jortner (MLJ) rate equation $^{52}$

$$
\begin{aligned}
k_{M L J}=\frac{2 \pi}{\hbar}\left|H_{S O C}\right|^{2} & \sqrt{\frac{1}{4 \pi k T \lambda}} \sum_{n} \frac{e^{-S} S^{n}}{n !} \\
& \times \exp \left(-\frac{\left(\lambda+n \hbar \omega_{e f f}+\Delta E\right)^{2}}{4 \lambda k T}\right),
\end{aligned}
$$

in which $\omega_{e f f}$ is an effective frequency representative of the nonclassical high frequency molecular modes and $S$ is the Huang-Rhys factor associated with this mode. Following previous work, we used typical values for the effective frequency and Huang-Rhys factor ${ }^{28}\left(\omega_{e f f}=1200 \mathrm{~cm}^{-1}, S=0.336\right)$. The remaining parameters are taken from calculations performed on the optimized $S_{1}$ and $T_{1}$ geometries in the cases of ISC and rISC, respectively. The other theoretical approach shown in Table I (denoted by Avg) consists in applying the MLJ rate 


\begin{tabular}{lllllll}
\hline \hline Molecule & Process & NEM & MLJ & Avg & Exp & Ref. \\
\hline \hline \multirow{2}{*}{ PTZ-DBTO2 } & $k_{I S C}\left(s^{-1}\right)$ & $1.6 \times 10^{8}$ & $2.3 \times 10^{8}$ & $1.9 \times 10^{8}$ & $1.0 \times 10^{7}$ & 42,43 \\
& $k_{r I S C}\left(s^{-1}\right)$ & $4.5 \times 10^{5}$ & $1.4 \times 10^{-26}$ & $5.4 \times 10^{4}$ & $1.4 \times 10^{6}$ & 42,43 \\
\hline \multirow{4}{*}{4 CzIPN } & $k_{I S C}\left(s^{-1}\right)$ & $5.8 \times 10^{7}$ & $8.2 \times 10^{7}$ & $1.2 \times 10^{8}$ & $5.1 \times 10^{7}$ & 44 \\
& $k_{r I S C}\left(s^{-1}\right)$ & $3.3 \times 10^{4}$ & $1.1 \times 10^{-1}$ & $1.5 \times 10^{4}$ & $2.7 \times 10^{6}$ & 44 \\
\hline \hline
\end{tabular}

TABLE I. Calculated intersystem crossing and reverse intersystem crossing rates for PTZ-DBTO2 and 4CzIPN with three different methods and the corresponding experimental values.

equation to all 500 sampled geometries and averaging the results.

Comparison between the various rates in Table I shows that for ISC, there is overall agreement between all the theoretical methods. The same cannot be said when considering the rISC process, for which the MLJ method is shown to predict near zero values. The averaging of the MLJ rates over the sampled geometries appears to resolve this issue, raising rISC rates. These, however, still fall short of the rISC rates calculated with the method developed in this work. More importantly, comparison between the theoretical methods and experimental results shows that the rates predicted by the our method are, in all cases, closer to the experimental values.

In the particular case of PTZ-DBTO2, the predicted ISC rate is roughly one order of magnitude greater than its experimental counterpart, while the calculated rISC rate underestimates the experimental value by a factor of 3 . These results also compare favorably with those from quantum dynamics calculations ${ }^{23}$, which, taking into account the vibronic coupling between two triplet states with localized and charge transfer character, produce an ISC rate of $5 \times 10^{5} \mathrm{~s}^{-1}$ and a rISC rate of $7 \times 10^{4} \mathrm{~s}^{-1}$. One rationalization for this fact is that the sampling procedure may result in the electronic character of the $T_{1}$ state to vary among conformations between localized and charge transfer states, effectively accounting for the nonadiabatic coupling.

For the 4CzIPN molecule, great agreement is found between predicted and experimental ISC rates. The calculated rISC rate, however, is seen to underestimate the experimental value by two orders of magnitude. This rate has also been the object of investigation in a previous work ${ }^{28}$, which, based on the MLJ rate expression, estimated it to lie in a range from $4.92 \times 10^{6}$ to $1.83 \times 10^{7}$. This was due to the very low singlet-triplet gap found $(0.01 \mathrm{eV})$, which resulted from a long range corrected functional tuning procedure performed with PCM. This procedure leads to very low values for the range separation parameter, which, in addition to producing low singlet-triplet gaps, also severely underestimates excitation energies ${ }^{53}$.

We may understand better the relationship between the different methods of calculation shown here by looking into the distribution of the two relevant parameters for (r)ISC rate calculations: singlet-triplet gaps and spin-orbit couplings. These distributions are shown in Figure 2 for each molecule and each excited state $\left(S_{1}\right.$ and $\left.T_{1}\right)$. It can be noted that in the case of PTZ-DBTO2 (Figure 2-a to d) the distributions for both fea- tures change considerably depending on the state from which geometries are sampled. In particular, the range of spin-orbit coupling values becomes much more expanded in the case of $T_{1}$ geometries. However, this is also followed by a displacement in the average values of the single-triplet gap towards higher energies, which helps explain the lower rISC rate. This is in contrast to the results for 4CzIPN (Figure 2-e to h), which present similar distributions for geometries from the two states. In this case, the offset between ISC and rISC predicted rates comes from the larger reorganization energies obtained for $4 \mathrm{CzIPN}(0.1-0.2 \mathrm{eV}$, as seen in Table II), an order of magnitude greater than those found for PTZ-DBTO2 $(0.025 \mathrm{eV})$.

The singlet-triplet gap distributions also explain the reason why MLJ rates seem to provide correct rates for ISC, but fail in the case of rISC. As seen in Figure 2-c and g, the gaps calculated for the optimized $S_{1}$ geometries (marked in the figure by the vertical dashed line) are situated at the left of the corresponding distributions. The opposite behavior is verified in the case of $T_{1}$ geometries (Figure 2-d and h), which has the optimized values closer to the average of the distributions. This discrepancy is not observed, however, in the distribution of spin-orbit couplings.

\begin{tabular}{lll}
\hline \hline Molecules & $S_{1} \rightarrow T_{1}$ & $T_{1} \rightarrow S_{1}$ \\
\hline \hline & & \\
PTZ-DBTO2 & 0.025 & 0.025 \\
\hline 4CzIPN & 0.172 & 0.125 \\
\hline
\end{tabular}

TABLE II. Reorganizations energies (eV) for the two processes relevant for ISC and rISC.

We now turn to the method's prediction concerning emission rates. The simulated fluorescence and phosphorescence spectra for both molecules are shown in Figure 3. In the case of PTZ-DBTO2, the fluorescence spectrum peaks at $454 \mathrm{~nm}$, being blue shifted with respect to the experimental spectrum in toluene ${ }^{42}$. In fact, the simulated spectrum resembles more the fluorescence spectrum of the donor moiety (PTZ). This can be explained by the $S_{1}$ state displaying a charge transfer character, which undergoes considerable stabilization in solution. In contrast, the simulation, captures the contributions around $400 \mathrm{~nm}$ from a localized singlet state observed in zeonex (which has a similar dielectric constant to toluene). Similarly, the experimental phosphorescence peak is found to be around $500 \mathrm{~nm}$ in zeonex, in agreement with the results seen in Figure 3-b. The better agreement results also from the 

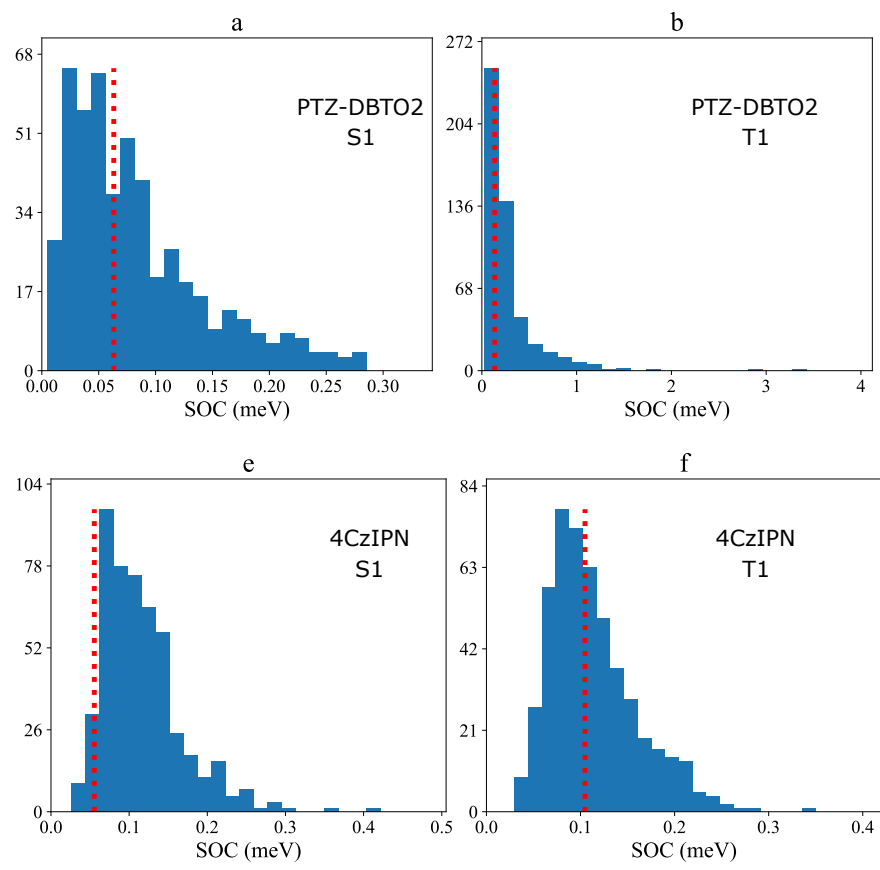
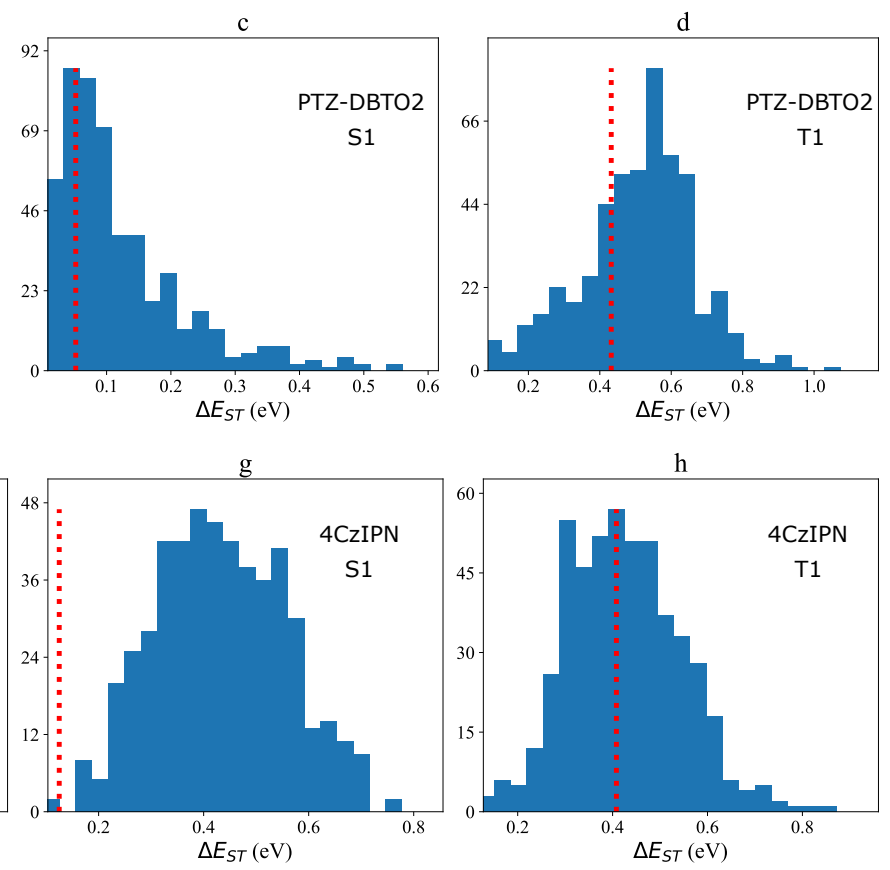

FIG. 2. Distribution of SOCs and singlet-triplet gaps for PTZ-DBTO2 (a to d) and 4CzIPN (e to f). Dashed lines correspond to values obtained in the corresponding equilibrium geometry.

localized character of the $T_{1}$ state, which manages to be well described by PCM calculations.

A similar situation is observed for $4 \mathrm{CZiPN}$ (Figure 3-c and d). The calculated fluorescence spectrum peaks at 436 $\mathrm{nm}$, whereas the experimental one peaks at around $500 \mathrm{~nm}$ in toluene ${ }^{44}$. The same issue concerning the charge transfer character of the $S_{1}$ state applies here. The calculated phosphorescence spectra for 4CzIPN is broad, with a peak at 516 $\mathrm{nm}$. The actual differential emission rate for phosphorescence, however, can be seen to be three orders of magnitude inferior to those found for PTZ-DBTO2, meaning that room temperature phosphorescence should not be observed for this molecule.

\begin{tabular}{lcccc}
\hline \hline Molecules & $k_{F}\left(s^{-1}\right)$ & $k_{P}\left(s^{-1}\right)$ & Exp. $k_{F}\left(s^{-1}\right)$ & Ref. \\
\hline \hline & & & & \\
PTZ-DBTO2 & $4.0 \times 10^{6}$ & $3.1 \times 10^{3}$ & $6.0 \times 10^{7}$ & 42,43 \\
\hline 4CzIPN & $9.3 \times 10^{7}$ & $1.7 \times 10^{1}$ & $1.7 \times 10^{7}$ & 44 \\
\hline
\end{tabular}

TABLE III. Calculated fluorescence $\left(k_{F}\right)$ and phosphorescence $\left(k_{P}\right)$ rates for PTZ-DBTO2 and 4CzIPN. Experimental fluorescence rates (Exp. $k_{F}$ ) are also shown.

By means of Equation 5, we are able to calculate the total fluorescence and phosphorescence rates for these molecules. Results are shown in Table III along with experimental estimates for fluorescence rates. It can be seen that calculated values show agreement with experiment within around one order of magnitude. Phosphorescence rates, in turn, are negligible for 4CzIPN, but are still significant for PTZ-DBTO2, as expected from the spectra.
Finally, we may investigate the joint effect of these various rates and processes in the kinetics of the population of the different states. To do so we resort to a set of differential equations governing the population of each state $\mathrm{s}^{43}$

$$
\begin{aligned}
\frac{d S_{1}}{d t} & =-\left(k_{F}+k_{I S C}+k_{n r}\right) S_{1}+k_{r I S C} T_{1} \\
\frac{d T_{1}}{d t} & =k_{I S C} S_{1}-\left(k_{P}+k_{r I S C}\right) T_{1} \\
\frac{d S_{0}}{d t} & =\left(k_{F}+k_{P}+k_{n r}\right) S_{1}
\end{aligned}
$$

where we have included a rate for non-radiative decay from the $S_{1}$ state $\left(k_{n r}\right)$. This follows an experimental estimation of a $1.5 \times 10^{6} \mathrm{~s}^{-1}$ non-radiative decay rate for $4 \mathrm{CzIPN}$ in toluene $^{44}$. In contrast, no significant non-radiative lost was observed for PTZ-DBTO2 solutions ${ }^{42}$.

The solution to these equations provide us with the timedependent photophysical behavior of the system. Figure 4-a and b show the temporal evolution of the $S_{1}$ and $T_{1}$ states of both molecules for an initial $S_{1}$ population of $100 \%$. In the case of PTZ-DBTO2, the initial depopulation of $S_{1}$ coincides with an increasing population of the $T_{1}$ state due to the high ISC rate in comparison to $k_{F}$. Low phosphorescence and rISC contribute to the maintenance of an $S_{1}$ population which decays again in a second moment, along with the triplet state. This behavior is in qualitative agreement with experimental results.

The dynamics in 4CzIPN, however, differs by a strong depopulation of $S_{1}$ following its high fluorescence rate. The triplet state experiences a less steep population change and 
a
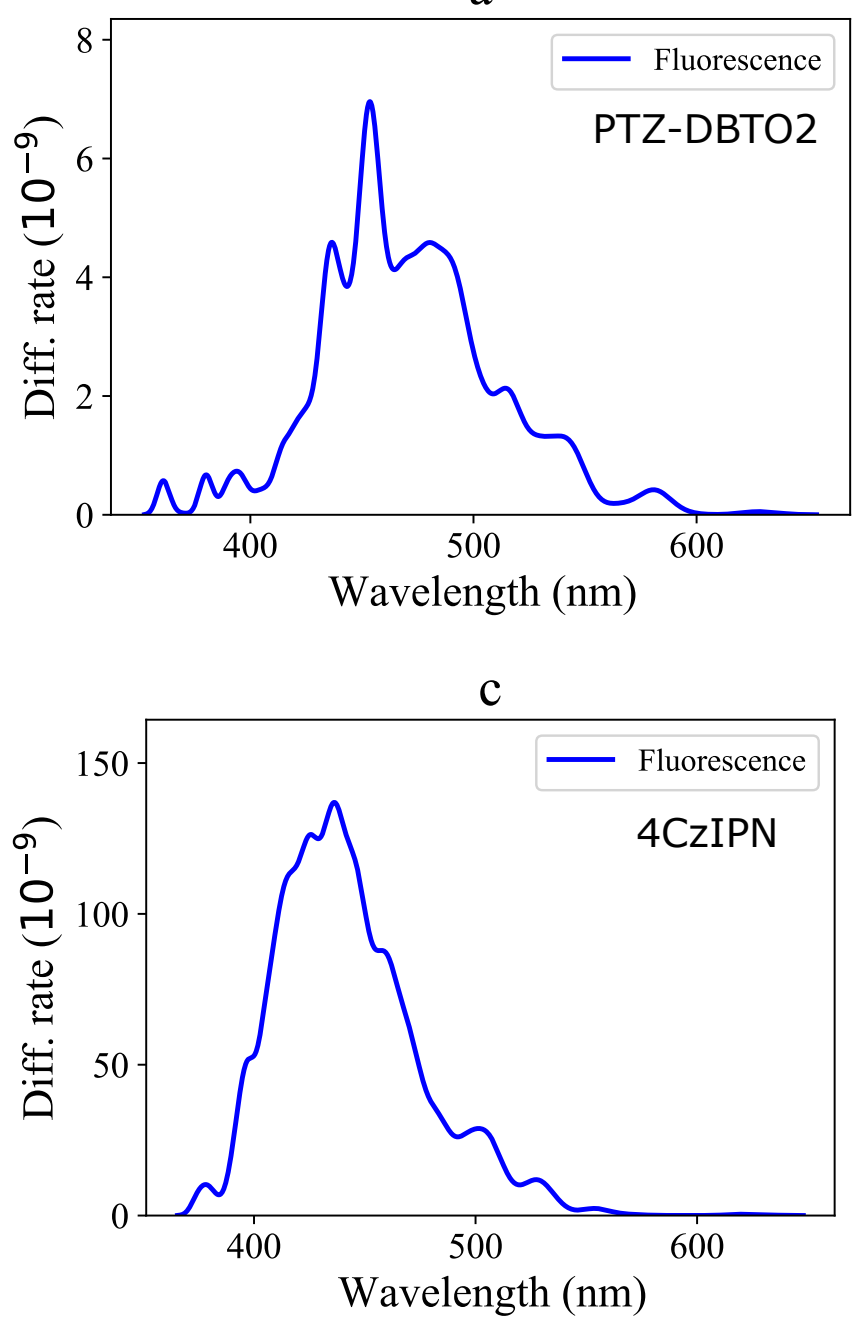

b
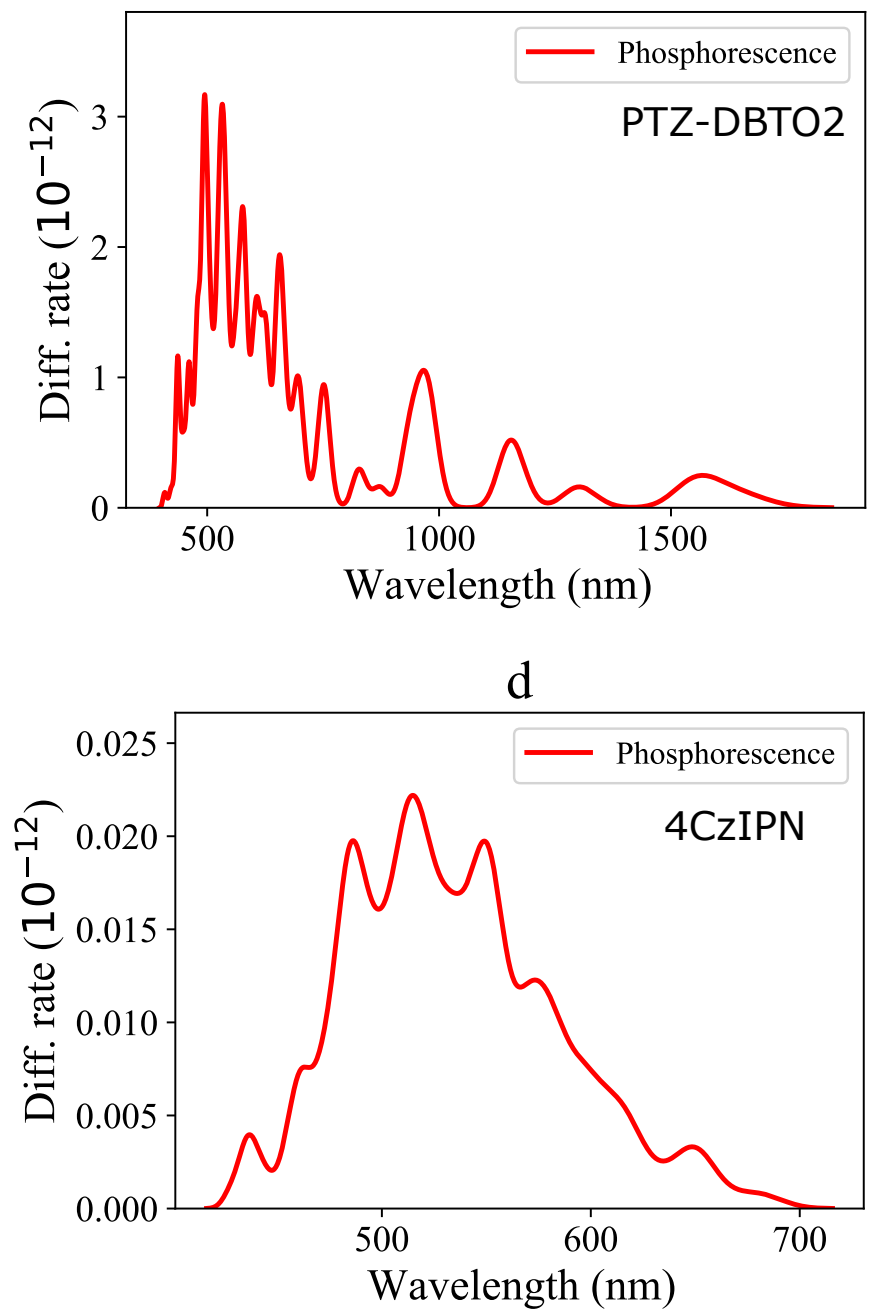

FIG. 3. Calculated fluorescence $(a, b)$ and phosphorescence $(c, d)$ emission spectra for PTZ-DBTO2 and 4CzIPN, respectively.

takes much longer to decrease again, as rISC rates are not particularly high and the phosphorescence channel is practically unavailable.

Comparison with experiment is made easier by considering the time-resolved photoluminescence decay, which can be simulated from the kinetic equations by splitting the $S_{0}$ state in three artificial states, each one being individually populated by fluorescence, phosphorescence and the non-radiative decay. In this sense, simulated time-resolved fluorescence and phosphorescence decays are shown in Figure 4-c and d for both molecules.

For PTZ-DBTO2, two aspects are noteworthy: first, the contribution from prompt fluorescence can be seen by the small vertical line in the initial times, followed by the delayed fluorescence that results from rISC. Second, we see significant contributions from phosphorescence (represented by the red curve). In fact, the calculations predict that $79 \%$ of the emission stems from fluorescence, with the remaining $21 \%$ being due to phosphorescence. Fitting the fluorescence curve with a biexponential function provides us with an estimated lifetime of $67 \mu$ s for the delayed component. This overestimates experimental lifetimes for the delayed component in about an order of magnitude, which is a result of the differences between calculated and experimental estimates for the rISC rate.

In contrast to these results, the $4 \mathrm{CzIPN}$ simulated timeresolved decay shows no contribution from phosphorescence, as expected. It does show a strong prompt component with a $6 \mathrm{~ns}$ lifetime, close to the experimental value of $14 \mathrm{~ns}$. The prompt intensity is 1.6 times higher than the delayed one, in qualitative agreement with experimental results. The delayed component, on the other hand, has an estimated lifetime of $45 \mu \mathrm{s}$, again overestimating the experimental value of almost $2 \mu$ s for the same reason as mentioned above in the case of PTZ-DBTO2. Interestingly, the simulated results appear to better match the experimental time-resolved decay produced at $200 \mathrm{~K}$, which displays much longer delayed lifetimes. One rationalization for this observation is the possibility that the 
a
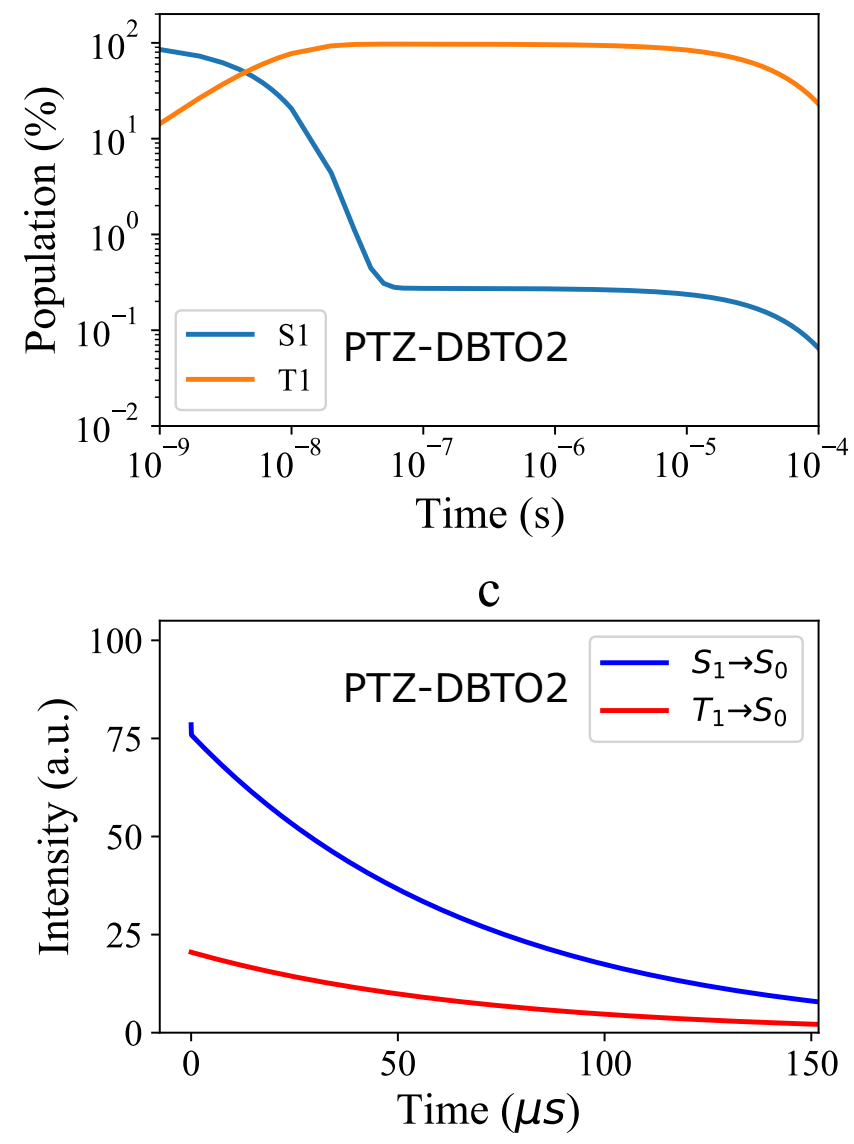

b
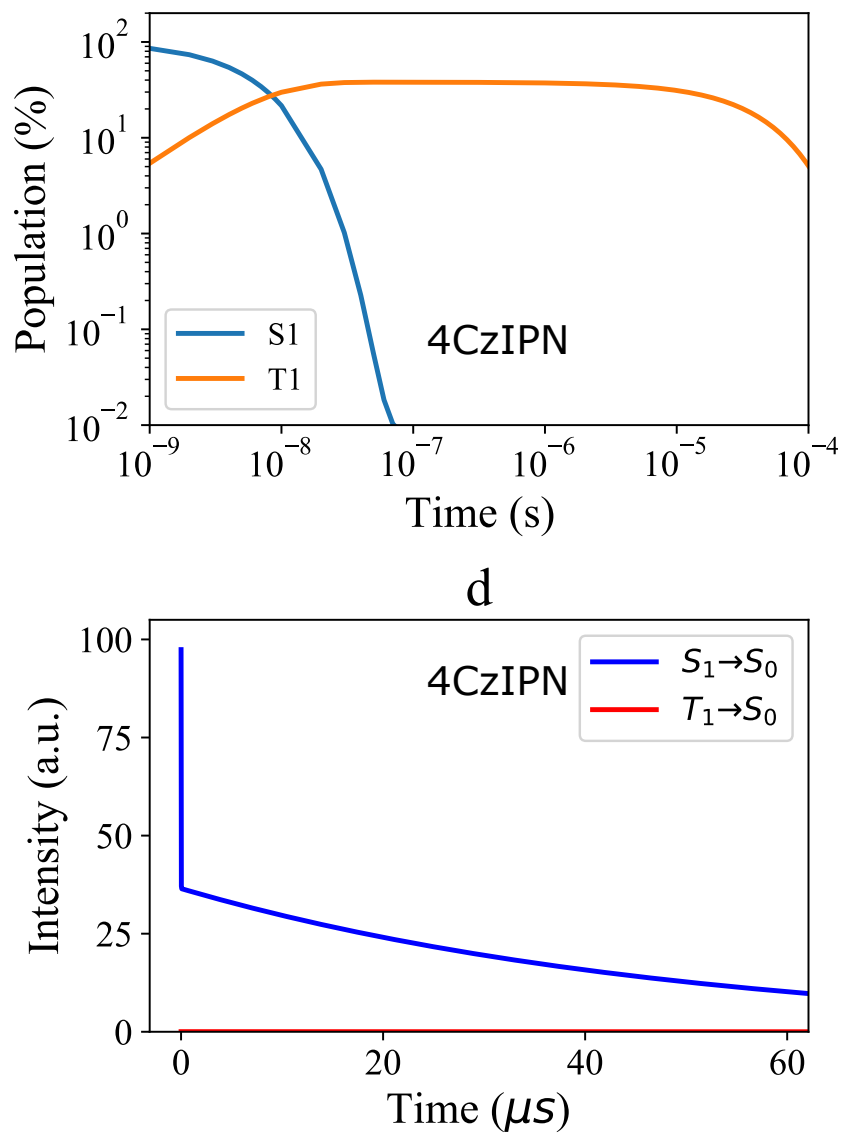

FIG. 4. a) Population occupation of S1 and T1 states as a function of time. b) Time-resolved photoluminescence decay of fluorescence and phosphorescence.

harmonic approximation that is behind the procedure of geometry sampling may overlook important anharmonic contributions that are relevant at higher temperatures, making results resemble more lower temperature experimental behavior. It is also worth mention that the non-radiative decay plays a minor role in the simulation, reducing the quantum yield in only $3 \%$, versus $6 \%$ in the experiment.

The overall results for the predicted time-dependent photophysical properties of these two molecules are very positive, especially by taking into account the fact that a precise agreement with experimental results requires the simultaneous precise estimation of four different rates. In this sense, it is clear that the method presented here is very powerful as it is able to, in a single framework, provide adequate qualitative and quantitative estimations for several properties of interest. Further improvement of the method can be achieved, for instance, by focusing on obtaining higher precision from the electronic structure calculations. The calculations of singlet-triplet gaps, i.e. the key parameter for TADF efficiency, has been shown to be sensitive to the electronic structure method ${ }^{54-56}$. Also, the results may be improved by considering state-specific solvation methods ${ }^{57,58}$ which better account for the stabilization of charge transfer states. Incidentally, if we were to offset our calculated 4CzIPN singlet energies by $0.3 \mathrm{eV}$, roughly the amount by which the simulated fluorescence spectra is blue shifted with respect to experiment, rISC rates would increase to $4.9 \times 10^{7} \mathrm{~s}^{-1}$, making it approach the experimental value. A similar calculation holds also for PTZ-DBTO2. This ad hoc shifting of energy levels, however, is a crude approximation, as it fails to take into account the possibility of geometries for which the $S_{1}$ state displays not a charge transfer character, but rather a localized one, in which case its energy should not be expected to change under solvation. As such, the distribution of singlet-triplet gaps is not only expected to shift, but also change in shape.

\section{CONCLUSIONS}

In summary, we have presented a theoretical approach that goes beyond the Condon approximation and is able to, within a unified framework, provide rate estimates for intersystem crossing, reverse intersystem crossing, fluorescence and phosphorescence. 
We show that the proposed method produces estimates in overall good agreement with experimental results, although it underestimates rISC rates due to an overestimation of singlet energies in solution. Results, however, compare favorably with the usually employed MLJ formula and also with other previous calculations available in the literature without the need to account explicitly for higher order effects such as vibronic couplings to higher lying excited states.

Once the rate estimates were obtained, we were able to show how these different processes affect the photophysical behavior. This was done by looking into the calculated time evolution of the population of singlet and triplet states and by comparing simulated time-resolved photoluminescence decay curves with experimental results. We observed that kinetics differed significantly for both molecules analyzed here. Whereas for PTZ-DBTO2 the delayed component of fluorescence is responsible for most of the luminescence, for 4CzIPN the prompt component has much higher intensity. Furthermore, we observed a significant contribution from phosphorescence for PTZ-DBTO2, which is not present in the results for 4 CzIPN. Inclusion of non-radiative decay rates also allowed the prediction of fluorescence quantum yields in agreement with experiments.

The method developed here may be further improved by increasing the accuracy of the electronic structure methods upon which it depends. We also observe that the underestimation of rISC rates and consequential overestimation of delayed fluorescence lifetimes leads to results that more closely resemble experiments performed at lower temperatures. This also suggests that improving geometry sampling beyond the harmonic approximation may partially correct this issue.

In conclusion, our approach may be valuable to the development of new TADF materials, providing a framework upon which all the processes of interest can be analyzed, quantified, and assembled together to provide predictions for actual photophysical behavior.

\section{ACKNOWLEDGMENTS}

The authors acknowledge support by a research grant (00028053) from VILLUM FONDEN.

\section{REFERENCES}

\footnotetext{
${ }^{1}$ M. Baldo, M. E. ThompSOn, and S. Forrest, "High-efficiency fluorescent organic light-emitting devices using a phosphorescent sensitizer," Nature 403, 750-753 (2000).

${ }^{2}$ Y. Sun, N. C. Giebink, H. Kanno, B. Ma, M. E. Thompson, and S. R. Forrest, "Management of singlet and triplet excitons for efficient white organic light-emitting devices," Nature 440, 908-912 (2006).

${ }^{3}$ K. T. Kamtekar, A. P. Monkman, and M. R. Bryce, "Recent advances in white organic light-emitting materials and devices (woleds)," Advanced Materials 22, 572-582 (2010).

${ }^{4}$ S. Reineke, F. Lindner, G. Schwartz, N. Seidler, K. Walzer, B. Lüssem, and K. Leo, "White organic light-emitting diodes with fluorescent tube efficiency," Nature 459, 234-238 (2009).

${ }^{5}$ M. Pope, H. Kallmann, and P. Magnante, "Electroluminescence in organic crystals,” The Journal of Chemical Physics 38, 2042-2043 (1963).
}

${ }^{6}$ S. Lamansky, P. Djurovich, D. Murphy, F. Abdel-Razzaq, H.-E. Lee, C. Adachi, P. E. Burrows, S. R. Forrest, and M. E. Thompson, "Highly phosphorescent bis-cyclometalated iridium complexes: synthesis, photophysical characterization, and use in organic light emitting diodes,' Journal of the American Chemical Society 123, 4304-4312 (2001).

${ }^{7}$ G. Zhou, W.-Y. Wong, and X. Yang, "New design tactics in oleds using functionalized 2-phenylpyridine-type cyclometalates of iridium (iii) and platinum (ii)," Chemistry-An Asian Journal 6, 1706-1727 (2011).

${ }^{8}$ S. Schmidbauer, A. Hohenleutner, and B. König, "Chemical degradation in organic light-emitting devices: Mechanisms and implications for the design of new materials," Advanced Materials 25, 2114-2129 (2013).

${ }^{9}$ S.-Y. Kim, W.-I. Jeong, C. Mayr, Y.-S. Park, K.-H. Kim, J.-H. Lee, C.K. Moon, W. Brütting, and J.-J. Kim, "Organic light-emitting diodes with $30 \%$ external quantum efficiency based on a horizontally oriented emitter," Advanced Functional Materials 23, 3896-3900 (2013).

${ }^{10}$ F. B. Dias, K. N. Bourdakos, V. Jankus, K. C. Moss, K. T. Kamtekar, V. Bhalla, J. Santos, M. R. Bryce, and A. P. Monkman, "Triplet harvesting with $100 \%$ efficiency by way of thermally activated delayed fluorescence in charge transfer oled emitters," Advanced Materials 25, 3707-3714 (2013).

${ }^{11}$ Y. Tao, K. Yuan, T. Chen, P. Xu, H. Li, R. Chen, C. Zheng, L. Zhang, and W. Huang, "Thermally activated delayed fluorescence materials towards the breakthrough of organoelectronics," Advanced materials 26, 7931-7958 (2014).

${ }^{12}$ H. Wang, L. Xie, Q. Peng, L. Meng, Y. Wang, Y. Yi, and P. Wang, "Novel thermally activated delayed fluorescence materials-thioxanthone derivatives and their applications for highly efficient oleds," Advanced Materials 26, 5198-5204 (2014).

${ }^{13}$ T.-A. Lin, T. Chatterjee, W.-L. Tsai, W.-K. Lee, M.-J. Wu, M. Jiao, K.-C. Pan, C.-L. Yi, C.-L. Chung, K.-T. Wong, et al., "Sky-blue organic light emitting diode with $37 \%$ external quantum efficiency using thermally activated delayed fluorescence from spiroacridine-triazine hybrid," Advanced Materials 28, 6976-6983 (2016).

${ }^{14}$ H. Nakanotani, T. Higuchi, T. Furukawa, K. Masui, K. Morimoto, M. Numata, H. Tanaka, Y. Sagara, T. Yasuda, and C. Adachi, "High-efficiency organic light-emitting diodes with fluorescent emitters," Nature communications 5, 1-7 (2014)

${ }^{15}$ H. Abroshan, V. Coropceanu, and J.-L. Bredas, "Hyperfluorescence-based emission in purely organic materials: Suppression of energy-loss mechanisms via alignment of triplet excited states," ACS Materials Letters 2, 1412-1418 (2020).

${ }^{16}$ H. Uoyama, K. Goushi, K. Shizu, H. Nomura, and C. Adachi, "Highly efficient organic light-emitting diodes from delayed fluorescence," Nature 492, 234-238 (2012).

${ }^{17}$ H. Sun, Z. Hu, C. Zhong, X. Chen, Z. Sun, and J.-L. Brédas, "Impact of dielectric constant on the singlet-triplet gap in thermally activated delayed fluorescence materials," The journal of physical chemistry letters 8, 2393 2398 (2017).

${ }^{18}$ R. Gómez-Bombarelli, J. Aguilera-Iparraguirre, T. D. Hirzel, D. Duvenaud, D. Maclaurin, M. A. Blood-Forsythe, H. S. Chae, M. Einzinger, D.-G. Ha, T. Wu, et al., "Design of efficient molecular organic light-emitting diodes by a high-throughput virtual screening and experimental approach," Nature materials 15, 1120-1127 (2016).

${ }^{19}$ Y. Shu and B. G. Levine, "Simulated evolution of fluorophores for light emitting diodes," The Journal of chemical physics 142, 104104 (2015).

${ }^{20}$ X. Zhang, W. Shen, D. Zhang, Y. Zheng, R. He, and M. Li, "Theoretical investigation of dihydroacridine and diphenylsulphone derivatives as thermally activated delayed fluorescence emitters for organic light-emitting diodes," RSC Advances 5, 51586-51591 (2015).

${ }^{21}$ J. S. Ward, R. S. Nobuyasu, A. S. Batsanov, P. Data, A. P. Monkman, F. B. Dias, and M. R. Bryce, "The interplay of thermally activated delayed fluorescence (tadf) and room temperature organic phosphorescence in sterically-constrained donor-acceptor charge-transfer molecules," Chemical Communications 52, 2612-2615 (2016).

${ }^{22}$ X.-K. Chen, S.-F. Zhang, J.-X. Fan, and A.-M. Ren, "Nature of highly efficient thermally activated delayed fluorescence in organic light-emitting diode emitters: nonadiabatic effect between excited states," The Journal of Physical Chemistry C 119, 9728-9733 (2015).

${ }^{23}$ J. Gibson, A. P. Monkman, and T. J. Penfold, "The importance of vibronic coupling for efficient reverse intersystem crossing in thermally activated delayed fluorescence molecules," ChemPhysChem 17, 2956 (2016). 
${ }^{24} \mathrm{C}$. M. Marian, "Mechanism of the triplet-to-singlet upconversion in the assistant dopant acrxtn," The Journal of Physical Chemistry C 120, 37153721 (2016).

${ }^{25}$ P. de Silva, C. A. Kim, T. Zhu, and T. Van Voorhis, "Extracting design principles for efficient thermally activated delayed fluorescence (tadf) from a simple four-state model," Chemistry of Materials 31, 6995-7006 (2019).

${ }^{26}$ C. M. Marian, "Spin-orbit coupling and intersystem crossing in molecules," Wiley Interdisciplinary Reviews: Computational Molecular Science 2, 187-203 (2012)

${ }^{27}$ T. J. Penfold, E. Gindensperger, C. Daniel, and C. M. Marian, "Spinvibronic mechanism for intersystem crossing," Chemical reviews 118, 6975-7025 (2018).

${ }^{28}$ P. K. Samanta, D. Kim, V. Coropceanu, and J.-L. Bredas, "Up-conversion intersystem crossing rates in organic emitters for thermally activated delayed fluorescence: impact of the nature of singlet vs triplet excited states," Journal of the American Chemical Society 139, 4042-4051 (2017).

${ }^{29}$ K. Schmidt, S. Brovelli, V. Coropceanu, D. Beljonne, J. Cornil, C. Bazzini, T. Caronna, R. Tubino, F. Meinardi, Z. Shuai, et al., "Intersystem crossing processes in nonplanar aromatic heterocyclic molecules," The Journal of Physical Chemistry A 111, 10490-10499 (2007).

${ }^{30} \mathrm{~J}$. Tatchen, N. Gilka, and C. M. Marian, "Intersystem crossing driven by vibronic spin-orbit coupling: a case study on psoralen," Physical chemistry chemical physics 9, 5209-5221 (2007).

${ }^{31}$ Q. Peng, Y. Niu, C. Deng, and Z. Shuai, "Vibration correlation function formalism of radiative and non-radiative rates for complex molecules," Chemical Physics 370, 215-222 (2010).

${ }^{32}$ R. Crespo-Otero and M. Barbatti, "Spectrum simulation and decomposition with nuclear ensemble: formal derivation and application to benzene, furan and 2-phenylfuran," in Marco Antonio Chaer Nascimento (Springer, 2014) pp. 89-102.

${ }^{33}$ L. E. de Sousa, L. A. Ribeiro, A. L. d. A. Fonseca, and D. A. da Silva Filho, "Modeling the emission spectra of organic molecules: A competition between franck-condon and nuclear ensemble methods," The Journal of Physical Chemistry A 120, 5380-5388 (2016).

${ }^{34}$ L. E. de Sousa, F. T. Bueno, G. M. e Silva, D. A. da Silva Filho, and P. H. de Oliveira Neto, "Fast predictions of exciton diffusion length in organic materials," Journal of Materials Chemistry C 7, 4066-4071 (2019).

${ }^{35}$ D. J. Tannor and E. J. Heller, "Polyatomic raman scattering for general harmonic potentials," The Journal of Chemical Physics 77, 202-218 (1982).

${ }^{36}$ R. Feynman, "Statistical mechanics: a set of lectures (advanced book classics)," (1998).

${ }^{37}$ Y. Niu, Q. Peng, C. Deng, X. Gao, and Z. Shuai, "Theory of excited state decays and optical spectra: application to polyatomic molecules," The Journal of Physical Chemistry A 114, 7817-7831 (2010).

${ }^{38}$ B. Minaev, G. Baryshnikov, and H. Agren, "Principles of phosphorescent organic light emitting devices," Physical Chemistry Chemical Physics 16, 1719-1758 (2014).

${ }^{39}$ J. Ulstrup and J. Jortner, "The effect of intramolecular quantum modes on free energy relationships for electron transfer reactions," The Journal of chemical physics 63, 4358-4368 (1975).

${ }^{40}$ E. R. Heller and J. O. Richardson, "Semiclassical instanton formulation of marcus-levich-jortner theory," The Journal of Chemical Physics 152, 244117 (2020).

${ }^{41}$ R. A. Marcus, "On the theory of oxidation-reduction reactions involving electron transfer. i," The Journal of chemical physics 24, 966-978 (1956).

${ }^{42}$ R. S. Nobuyasu, Z. Ren, G. C. Griffiths, A. S. Batsanov, P. Data, S. Yan, A. P. Monkman, M. R. Bryce, and F. B. Dias, "Rational design of tadf polymers using a donor-acceptor monomer with enhanced tadf efficiency induced by the energy alignment of charge transfer and local triplet excited states," Advanced Optical Materials 4, 597-607 (2016).

${ }^{43}$ T. Penfold, F. Dias, and A. P. Monkman, "The theory of thermally activated delayed fluorescence for organic light emitting diodes," Chemical communications 54, 3926-3935 (2018).
${ }^{44}$ R. Ishimatsu, S. Matsunami, K. Shizu, C. Adachi, K. Nakano, and T. Imato, "Solvent effect on thermally activated delayed fluorescence by 1, 2, 3, 5-tetrakis (carbazol-9-yl)-4, 6-dicyanobenzene," The Journal of Physical Chemistry A 117, 5607-5612 (2013).

${ }^{45} \mathrm{~S}$. Hirata and M. Head-Gordon, "Time-dependent density functional theory within the tamm-dancoff approximation," Chemical Physics Letters 314, 291-299 (1999).

${ }^{46}$ M. J. Peach, M. J. Williamson, and D. J. Tozer, "Influence of triplet instabilities in tddft," Journal of chemical theory and computation 7, 3578-3585 (2011).

${ }^{47}$ A. Chantzis, A. D. Laurent, C. Adamo, and D. Jacquemin, "Is the tammdancoff approximation reliable for the calculation of absorption and fluorescence band shapes?" Journal of chemical theory and computation $\mathbf{9}$, 4517-4525 (2013).

${ }^{48} \mathrm{G}$. Scalmani and M. J. Frisch, "Continuous surface charge polarizable continuum models of solvation. i. general formalism," The Journal of chemical physics 132, 114110 (2010).

${ }^{49}$ Y. Zhao and D. G. Truhlar, "The m06 suite of density functionals for main group thermochemistry, thermochemical kinetics, noncovalent interactions, excited states, and transition elements: two new functionals and systematic testing of four m06-class functionals and 12 other functionals," Theoretical chemistry accounts 120, 215-241 (2008).

${ }^{50}$ M. J. Frisch, G. W. Trucks, H. B. Schlegel, G. E. Scuseria, M. A. Robb, J. R. Cheeseman, G. Scalmani, V. Barone, G. A. Petersson, H. Nakatsuji, X. Li, M. Caricato, A. V. Marenich, J. Bloino, B. G. Janesko, R. Gomperts, B. Mennucci, H. P. Hratchian, J. V. Ortiz, A. F. Izmaylov, J. L. Sonnenberg, D. Williams-Young, F. Ding, F. Lipparini, F. Egidi, J. Goings, B. Peng, A. Petrone, T. Henderson, D. Ranasinghe, V. G. Zakrzewski, J. Gao, N. Rega, G. Zheng, W. Liang, M. Hada, M. Ehara, K. Toyota, R. Fukuda, J. Hasegawa, M. Ishida, T. Nakajima, Y. Honda, O. Kitao, H. Nakai, T. Vreven, K. Throssell, J. A. Montgomery, Jr., J. E. Peralta, F. Ogliaro, M. J. Bearpark, J. J. Heyd, E. N. Brothers, K. N. Kudin, V. N. Staroverov, T. A. Keith, R. Kobayashi, J. Normand, K. Raghavachari, A. P. Rendell, J. C. Burant, S. S. Iyengar, J. Tomasi, M. Cossi, J. M. Millam, M. Klene, C. Adamo, R. Cammi, J. W. Ochterski, R. L. Martin, K. Morokuma, O. Farkas, J. B. Foresman, and D. J. Fox, "Gaussian 16 Revision C.01," (2016), gaussian Inc. Wallingford CT.

${ }^{51}$ Y. Shao, Z. Gan, E. Epifanovsky, A. T. Gilbert, M. Wormit, J. Kussmann, A. W. Lange, A. Behn, J. Deng, X. Feng, et al., "Advances in molecular quantum chemistry contained in the q-chem 4 program package," Molecular Physics 113, 184-215 (2015).

${ }^{52}$ J. Jortner, "Temperature dependent activation energy for electron transfer between biological molecules," The Journal of Chemical Physics 64, 48604867 (1976).

${ }^{53}$ L. Kronik and S. Kümmel, "Dielectric screening meets optimally tuned density functionals," Advanced Materials 30, 1706560 (2018).

${ }^{54}$ H. Sun, C. Zhong, and J.-L. Bredas, "Reliable prediction with tuned rangeseparated functionals of the singlet-triplet gap in organic emitters for thermally activated delayed fluorescence," Journal of chemical theory and computation 11, 3851-3858 (2015).

${ }^{55}$ S. Huang, Q. Zhang, Y. Shiota, T. Nakagawa, K. Kuwabara, K. Yoshizawa, and C. Adachi, "Computational prediction for singlet-and triplet-transition energies of charge-transfer compounds," Journal of chemical theory and computation 9, 3872-3877 (2013).

${ }^{56} \mathrm{P}$. de Silva, "Inverted singlet-triplet gaps and their relevance to thermally activated delayed fluorescence," The journal of physical chemistry letters 10, 5674-5679 (2019).

${ }^{57}$ M. Caricato, B. Mennucci, J. Tomasi, F. Ingrosso, R. Cammi, S. Corni, and G. Scalmani, "Formation and relaxation of excited states in solution: A new time dependent polarizable continuum model based on time dependent density functional theory," The Journal of chemical physics 124, 124520 (2006).

${ }^{58}$ C. A. Guido, D. Jacquemin, C. Adamo, and B. Mennucci, "Electronic excitations in solution: the interplay between state specific approaches and a time-dependent density functional theory description," Journal of chemical theory and computation 11, 5782-5790 (2015). 